\title{
Operation-induced decoherence by nonrelativistic scattering from a quantum memory
}

\author{
D Margetis ${ }^{1}$ and J M Myers ${ }^{2}$ \\ ${ }^{1}$ Department of Mathematics, and Institute for Physical Science and Technology, \\ University of Maryland, College Park, MD 20742, USA \\ 2 Division of Engineering and Applied Sciences, Harvard University, Cambridge, MA 02138, \\ USA \\ E-mail: dio@math.umd.edu and myers@deas.harvard.edu
}

Received 16 June 2006, in final form 7 August 2006

Published 29 August 2006

Online at stacks.iop.org/JPhysA/39/11567

\begin{abstract}
Quantum computing involves transforming the state of a quantum memory. We view this operation as performed by transmitting nonrelativistic (massive) particles that scatter from the memory. By using a system of $(1+1)$ dimensional, coupled Schrödinger equations with point interaction and narrowband incoming pulse wavefunctions, we show how the outgoing pulse becomes entangled with a two-state memory. This effect necessarily induces decoherence, i.e., deviations of the memory content from a pure state. We describe incoming pulses that minimize this decoherence effect under a constraint on the duration of their interaction with the memory.
\end{abstract}

PACS numbers: $03.65 .-\mathrm{w}, 03.67 .-\mathrm{a}, 03.65 . \mathrm{Nk}, 03.65 . \mathrm{Yz}, 03.67 . \mathrm{Lx}$

\section{Introduction}

Quantum computing [1-4] relies on a sequence of operations, each of which transforms a pure quantum state to, ideally, another pure state. These states describe a physical system, usually called memory. A central problem in quantum computing is decoherence, in which the pure state degrades to a mixed state, with deleterious effects for the computation.

In the earliest discussions of quantum computing [1] the spatial variables are retained, although not in the form of the Schrödinger equation. Later studies [2, 4, 5] abandon the spatial variables: The Schrödinger equation that describes the state-transforming operation is simplified to an equation in which only the time derivative appears; the quantum memory consists of spins, or their generalizations, and computations are expressed as carried out by unitary transforms acting on memory states. In this context decoherence is attributed to 
extraneous influences unconnected with operations on the memory; it has been suggested that speeding the operations decreases decoherence $[6,7]$.

In a sequence of papers [8-11] the quantum memory is studied by inclusion of the spatial variables. Essential in this formulation is scattering: the memory content is transformed by scattering an outside signal from it; this signal can be one or several particles. The incoming and outgoing wavefunctions of the signal, $\psi^{\text {in }}(\mathbf{r})$ and $\psi^{\text {out }}(\mathbf{r})$, carry out important information about the memory [9]. While the combined system of memory and particles is subject to unitary transforms, the memory itself transforms unitarily only in the case of 'admissible' incoming wavefunctions; in one space dimension such waves are shown to be of single momentum [9]. The incoming wavefunction couples with the memory states through a localized interaction potential. For interaction at one point, the appropriate pseudo-potential in one space dimension is developed in [8] for the time-independent Schrödinger equation.

By this approach $[8,9]$ additional decoherence is connected specifically with the memory operations: for non-admissible, pulsed, incoming wavefunctions, decoherence results via tracing out the space variables. This effect is described in [9] explicitly for a two-state memory by use of the time-dependent, relativistic Schrödinger equation in one space dimension. In this case there is no dispersion and, thus, the time length of the incoming and outgoing pulses is well defined. It is found [9] that light pulses of given duration and carrier frequency minimize decoherence if they have a half-cycle cosine envelope, regardless of the details of the interaction potential. In this context speeding the memory operations by using shorter pulses increases decoherence.

In this paper we apply the general approach based on scattering [8-11] to study in detail the decoherence caused by nonrelativistic (hence massive) particles scattered from a twostate memory. For this purpose, we invoke the time-dependent, nonrelativistic Schrödinger equation with two coupled channels $[9,12]$ in one space dimension, and the pseudo-potential derived in [8]. Because the incoming wavefunction disperses, our results here differ from those for light scattering [9]. We define the pulse duration as the average time during which the particle wavefunction interacts with the memory at one point. We find that, by contrast to [9], the incident pulse of fixed duration that minimizes decoherence has a Gaussian envelope.

The scattering-based approach to quantum memory is motivated by the broader question of how to implement the ideas of quantum computing in the laboratory setting. So far as we are aware controlling the state of a memory requires probing it with photon or massive-particle beams. Probing involves scattering that usually entangles the memory with the probe, resulting in a memory state that is mixed rather than pure. This complication, noticed previously [13] in connection to nuclear magnetic resonance, causes decoherence directly by operations that aim to transform the memory states. This effect has been described in detail when the memory is probed with light [9]; but, to our knowledge, operation-induced decoherence has not been previously quantified when the memory is probed with massive particles such as electrons. Here we develop this latter scenario in detail, and describe the incoming particle beam that induces minimal decoherence.

To model operation-induced decoherence we analyse a two-state memory by using the pseudo-potential of $\mathrm{Wu}$ and $\mathrm{Yu}$ [8] in a system of two coupled Schrödinger equations in one space dimension. The two-component field of the scattering process for the combined particle-memory system is denoted $\Psi(x, t)$; this field is a two-element column vector represented by

$$
\Psi(x, t)=\left[\begin{array}{l}
\Psi_{1}(x, t) \\
\Psi_{2}(x, t)
\end{array}\right],
$$


where $\Psi_{j}(x, t)(j=1,2)$ are scalar functions. The reduced density matrix, $\rho$, for the memory is the $2 \times 2$ matrix

$$
\begin{aligned}
\rho(t) & :=\int_{-\infty}^{\infty} \mathrm{d} x \Psi(x, t) \Psi^{\dagger}(x, t) \\
& =\int_{-\infty}^{\infty} \mathrm{d} x\left[\begin{array}{cc}
\left|\Psi_{1}(x, t)\right|^{2} & \Psi_{1}(x, t) \Psi_{2}^{*}(x, t) \\
\Psi_{2}(x, t) \Psi_{1}^{*}(x, t) & \left|\Psi_{2}(x, t)\right|^{2}
\end{array}\right], \quad M:=\lim _{t \rightarrow \infty} \rho(t),
\end{aligned}
$$

which is obtained by tracing out the spatial variables. In the above equations, $x$ is the onedimensional space variable, $t$ is time, $\Psi^{\dagger}$ denotes the Hermitian conjugate of $\Psi, \Psi_{j}^{*}$ denotes the complex conjugate of $\Psi_{j}$, and $M$ corresponds to the final memory state; for a pure final state, $M^{2}=M$ and $\operatorname{tr}\left(M^{2}\right)=1$. Equation (2) follows closely equation (3) in [9]. We define the memory 'impurity' by

$$
\operatorname{Imp}(M):=\left[1-\operatorname{tr}\left(M^{2}\right)\right]^{1 / 2},
$$

where the square root is taken to achieve a desired scaling property, as explained in section 3. Equation (3) differs from the impurity used in [9], where $\operatorname{Imp}(M):=2\left[1-\operatorname{tr}\left(M^{2}\right)\right]$. We study the behaviour of $\operatorname{Imp}(M)$ for incoming wavefunctions that are characterized by the average time $T$ of their interaction with the memory at one point $(x=0)$, and carrier frequency $\omega_{0}$ where $\omega_{0} T \gg 1$; and quantify how the pulse envelope shape affects $\operatorname{Imp}(M)$. We show that as $\omega_{0} T \gg 1$, when the incoming wavefunction tends to obtain a definite momentum and become admissible [9], $\operatorname{Imp}(M)$ approaches zero not faster than $O\left(T^{-1}\right)$; the pulse envelope shape for the minimum $\operatorname{Imp}(M)$ is a Gaussian. This result is to be contrasted with the pulse wavefunction of half-cycle cosine envelope found in [9].

Our approach, based on scattering [9], should not be confused with a previous theory that uses 'continuous variables' [14]. The latter theory invokes a large number, $d$, of discrete states. In the limit $d \rightarrow \infty$ a description is adopted that resembles the use of continuous position and conjugate momentum or quantum field variables but without scattering.

It is beyond the scope of this paper to provide an exhaustive bibliography on quantum computing. The literature on this subject is vast and growing. The interested reader is referred to recent reviews $[6,7]$ that explore this body of work.

This paper is organized as follows. In section 2 we formulate the problem of decoherence for a quantum memory as a scattering problem with two coupled channels in one space dimension: In section 2.1 we restrict attention to symmetric (even) wavefunctions; and in section 2.2 we study the case with antisymmetric (odd) wavefunctions. In section 3 we apply approximations for sufficiently long pulse envelopes. In section 4 we minimize impurity under a constraint on the incoming pulse duration. In section 5 we summarize our results, and discuss a generalization and related, open problems. Throughout this paper we apply units with $\hbar^{2} /(2 m)=1$ where $m$ is the particle mass.

\section{Formulation}

In this section we describe the theoretical framework needed for calculating the impurity, $\operatorname{Imp}(M)$, defined by (3). We introduce the requisite equations of motion using a pseudopotential in one space dimension [8] for even and odd incoming wavefunctions; and derive formulae for the reduced density matrix $\rho(t)$ and its limit, $M$, in view of (2).

We consider a quantum memory with two states [8] that scatters massive particles in one space dimension. The two-component field of the particle-memory system is represented by 
the column vector (1) where $-\infty<x, t<+\infty$. The natural condition is imposed that this $\Psi$ be square integrable,

$$
\int_{-\infty}^{\infty} \mathrm{d} x \Psi^{\dagger}(x, t) \Psi(x, t)=\int_{-\infty}^{\infty} \mathrm{d} x\left[\left|\Psi_{1}(x, t)\right|^{2}+\left|\Psi_{2}(x, t)\right|^{2}\right]<\infty .
$$

Next, we describe the equation of motion for $\Psi(x, t)$. In one space dimension, this field solves the Schrödinger equation in the form [8]

$$
-\partial_{x x} \Psi(x, t)+\int_{-\infty}^{\infty} \mathrm{d} x^{\prime} V\left(x, x^{\prime}\right) \Psi\left(x^{\prime}, t\right)=\mathrm{i} \partial_{t} \Psi(x, t),
$$

where $\partial_{w}:=\partial / \partial w$ for $w=x, t$ and $V\left(x, x^{\prime}\right)$ is a $2 \times 2$ potential. For the quantum memory this potential $V$ is chosen to be the point interaction [8]

$$
V\left(x, x^{\prime}\right)=g_{3} \sigma_{3} \delta_{p}^{\prime}(x) \delta_{p}^{\prime}\left(x^{\prime}\right)+g_{1} \sigma_{1} \delta(x) \delta\left(x^{\prime}\right),
$$

where $\sigma_{1}$ and $\sigma_{3}$ are Pauli matrices; $\sigma_{3}=\operatorname{diag}(1,-1), \sigma_{1}=\Lambda \cdot \sigma_{3} \cdot \Lambda$, and

$$
\Lambda:=2^{-1 / 2}\left[\begin{array}{cc}
1 & 1 \\
1 & -1
\end{array}\right], \quad \Lambda^{2}=1
$$

The symbol $\delta_{p}^{\prime}(x)$ in (6) denotes $\delta^{\prime}(x)$ modified to remove any discontinuity at $x=0$ from the function on which it operates [8]: $\delta_{p}^{\prime}(x) g(x):=\delta^{\prime}(x)\left[1-\lim _{x \rightarrow 0^{+}}\right] g(x)$ for $x>0$ and $\delta_{p}^{\prime}(x) g(x):=\delta^{\prime}(x)\left[1-\lim _{x \rightarrow 0^{-}}\right] g(x)$ for $x<0$.

We now simplify (5) by introducing the symmetric and antisymmetric parts of $\Psi$,

$$
\begin{aligned}
& \Psi_{\mathrm{s}}(x, t):=\frac{1}{2}[\Psi(x, t)+\Psi(-x, t)], \\
& \Psi_{\mathrm{a}}(x, t):=\frac{1}{2}[\Psi(x, t)-\Psi(-x, t)], \quad \Psi=\Psi_{\mathrm{s}}+\Psi_{\mathrm{a}} .
\end{aligned}
$$

By (5) and (6), the $g_{1}$ and $g_{3}$ terms of $V$ apply separately to $\Psi_{\mathrm{s}}$ and $\Psi_{\mathrm{a}}$,

$$
\begin{aligned}
& -\partial_{x x} \Psi_{\mathrm{s}}(x, t)+g_{1} \sigma_{1} \delta(x) \Psi_{\mathrm{s}}(0, t)=\mathrm{i} \partial_{t} \Psi_{\mathrm{s}}(x, t), \\
& -\partial_{x x} \Psi_{\mathrm{a}}(x, t)-g_{3} \sigma_{3} \delta_{p}^{\prime}(x) \Psi_{\mathrm{a}}^{\prime}(0, t)=\mathrm{i} \partial_{t} \Psi_{\mathrm{a}}(x, t),
\end{aligned}
$$

where $\Psi_{\mathrm{a}}^{\prime}(0, t):=\lim _{x \rightarrow 0^{ \pm}} \partial_{x} \Psi_{\mathrm{a}}(x, t)$. Because $\sigma_{3}$ is diagonal, by (10) the scalar components $\Psi_{\mathrm{a} j}$ of $\Psi_{\mathrm{a}}$ decouple; by contrast, $\Psi_{\mathrm{s} j}$ are coupled from (9). To decouple $\Psi_{\mathrm{s} j}$ we define the two-component field $\Phi(x, t)$ by

$$
\Phi(x, t)=\left[\begin{array}{l}
\Phi_{1}(x, t) \\
\Phi_{2}(x, t)
\end{array}\right]:=\Lambda \cdot \Psi_{\mathrm{s}}(x, t) .
$$

From (9) and the definition of $\Phi$ we obtain

$$
-\partial_{x x} \Phi(x, t)+g_{1} \sigma_{3} \int_{\infty}^{\infty} \mathrm{d} x^{\prime} \delta(x) \delta\left(x^{\prime}\right) \Phi\left(x^{\prime}, t\right)=\mathrm{i} \partial_{t} \Phi(x, t) .
$$

So, for the symmetric part of $\Psi$, the decoupled equations for the components $\Phi_{j}(x, t)$ are

$$
-\partial_{x x} \Phi_{j}(x, t)+g_{1 j} \delta(x) \Phi_{j}(0, t)=\mathrm{i} \partial_{t} \Phi_{j}(x, t), \quad j=1,2,
$$

where

$$
g_{11}:=g_{1}=:-g_{12}
$$

For the antisymmetric part we have

$$
-\partial_{x x} \Psi_{\mathrm{a} j}(x, t)-g_{3 j} \delta_{p}^{\prime}(x) \Psi_{\mathrm{a} j}^{\prime}(0, t)=\mathrm{i} \partial_{t} \Psi_{\mathrm{a} j}(x, t),
$$

where

$$
g_{31}:=g_{3}=:-g_{32} \text {. }
$$


We assume that initially $(t \rightarrow-\infty)$ the quantum memory is at an arbitrary state $\left[c_{1}, c_{2}\right]^{\mathrm{T}}$ with $\left|c_{1}\right|^{2}+\left|c_{2}\right|^{2}=1$, where the $C^{\mathrm{T}}$ denotes the transpose of $C$; and the system particlememory forms the tensor product

$$
\Psi(x, t) \sim\left[\begin{array}{l}
c_{1} \\
c_{2}
\end{array}\right] \psi^{\text {in }}(x, t) \quad t \rightarrow-\infty,
$$

where $\psi^{\text {in }}(x, t)$ is the incoming particle wavefunction to be discussed in more detail below.

Before we proceed to analyse (13) and (15) with (17) we add a few comments on the reduced density matrices $\rho$ and $M$ defined by (2). The eigenvalues $\lambda_{1}$ and $\lambda_{2}$ of any $2 \times 2$ density matrix are nonnegative and obey $\lambda_{1}+\lambda_{2}=1$. It follows that

$$
1-\operatorname{tr}\left(\rho^{2}\right)=1-\lambda_{1}^{2}-\lambda_{2}^{2}=2 \lambda_{1}\left(1-\lambda_{1}\right)=2 \lambda_{1} \lambda_{2}=2 \operatorname{det} \rho .
$$

In particular, by (3) we obtain

$$
\operatorname{Imp}(M)=(2 \operatorname{det} M)^{1 / 2} .
$$

\subsection{Symmetric case}

In this subsection we turn our attention to symmetric wavefunctions setting $\Psi_{\mathrm{a}}(x, t) \equiv 0$, so that $\Psi \equiv \Psi_{\mathrm{s}}=\Lambda \cdot \Phi$ : we solve (13) with (17) for the scalar components $\Phi_{j}(x, t)$; and we derive a formula for the impurity $\operatorname{Imp}(M)$ in terms of the Fourier transform in time of the incoming wavefunction at $x=0$.

First, we determine $\Phi_{j}(x, t)$. By (17) we define the starting memory state

$$
\left[\begin{array}{l}
d_{1} \\
d_{2}
\end{array}\right]:=\Lambda \cdot\left[\begin{array}{l}
c_{1} \\
c_{2}
\end{array}\right] \text {. }
$$

Given the initial condition (17), where $\Psi$ and $c_{j}$ are replaced by $\Phi$ and $d_{j}$, we solve (13) by taking the Fourier transform of $\Phi_{j}$ in time; see appendix for details. We find

$$
\begin{aligned}
& \Phi_{j}(x, t)=d_{j} \int_{0}^{\infty} \frac{\mathrm{d} \omega}{\sqrt{2 \pi}} \mathrm{e}^{-\mathrm{i} \omega t} f(\omega)\left[\mathrm{e}^{-\mathrm{i} \sqrt{\omega}|x|}+S_{j}(\omega) \mathrm{e}^{\mathrm{i} \sqrt{\omega}|x|}\right], \\
& S_{j}(\omega):=\frac{2 \mathrm{i} \sqrt{\omega}+g_{1 j}}{2 \mathrm{i} \sqrt{\omega}-g_{1 j}}, \quad j=1,2,
\end{aligned}
$$

where only positive frequencies $\omega$ are considered as explained below; $f(\omega)$ is reasonably arbitrary except for satisfying (28) below.

Next, we derive a normalization condition for $f(\omega)$. In the limit $t \rightarrow-\infty$ the leadingorder contribution to integration in (21) comes from the $\mathrm{e}^{-\mathrm{i} \sqrt{\omega}|x|}$ term; hence,

$$
\Phi(x, t) \sim\left[\begin{array}{l}
d_{1} \\
d_{2}
\end{array}\right] \psi^{\text {in }}, \quad \psi^{\text {in }}(x, t) \equiv \int_{0}^{\infty} \frac{\mathrm{d} \omega}{\sqrt{2 \pi}} \mathrm{e}^{-\mathrm{i} \omega t} f(\omega) \mathrm{e}^{-\mathrm{i} \sqrt{\omega}|x|},
$$

which is the desired initial condition by (17) with (20). Equation (23) yields a normalization condition for $f(\omega)$ via the requirement

$$
\int_{-\infty}^{\infty} \mathrm{d} x\left|\psi^{\text {in }}(x, t)\right|^{2}=1 .
$$

More precisely, we define

$$
\psi^{+}(x, t):=\int_{0}^{\infty} \frac{\mathrm{d} \omega}{\sqrt{2 \pi}} f(\omega) \exp [-\mathrm{i}(\omega t-\sqrt{\omega} x)],
$$


and notice that, for negative and large enough $t, \psi^{+}(x, t)$ tends to zero except over a region of large, positive $x$. Thus, from (23) and (25),

$$
\psi^{\text {in }}(x, t) \sim \psi^{+}(x, t)+\psi^{+}(-x, t) \quad t \rightarrow-\infty,
$$

where there is negligible overlap between the two terms on the right-hand side of this equation. Thus, by (24) and (26),

$$
2 \int_{-\infty}^{\infty} \mathrm{d} x\left|\psi^{+}(x, t)\right|^{2}=1 .
$$

The substitution of (25) into (27) yields

$$
4 \int_{0}^{\infty} \mathrm{d} \omega \sqrt{\omega}|f(\omega)|^{2}=1
$$

We proceed to derive a formula for the impurity, $\operatorname{Imp}(M)$, in terms of $f(\omega)$. By (2) with (11) the reduced density matrix becomes

$$
\rho(t)=\Lambda \cdot\left(\int_{-\infty}^{\infty} \mathrm{d} x \Phi(x, t) \Phi^{\dagger}(x, t)\right) \cdot \Lambda .
$$

The use of $\Lambda$ leaves the determinant of $\rho$ unaltered; thus, by (19) with $M=\lim _{t \rightarrow \infty} \rho(t)$,

$$
\begin{aligned}
& \operatorname{Imp}(M)=\left(2 \lim _{t \rightarrow \infty} \operatorname{det}[\chi(t)]\right)^{1 / 2}, \\
& \chi(t):=\int_{-\infty}^{\infty} \mathrm{d} x \Phi(x, t) \Phi^{\dagger}(x, t) .
\end{aligned}
$$

We now express $\chi(t)$ in terms of $f(\omega)$ for large, positive $t$. By differentiating both sides of (31) and using (12) we obtain

$$
\dot{\chi}_{j k}(t)=\mathrm{i}\left(g_{1 k}-g_{1 j}\right) \Phi_{j}(0, t) \Phi_{k}^{*}(0, t), \quad j, k=1,2,
$$

where $g_{1 j}$ are defined by (14), the dot denotes time derivative, and the asterisk denotes complex conjugation. By (31) with (17), (20) and (24), $\operatorname{det}[\chi(t)]$ vanishes when $t \rightarrow-\infty$, as it should; in particular,

$$
\lim _{t \rightarrow-\infty} \chi(t)=\left[\begin{array}{l}
d_{1} \\
d_{2}
\end{array}\right]\left[d_{1}^{*}, d_{2}^{*}\right]
$$

To determine $\chi(t)$ as $t \rightarrow \infty$, we integrate (32) in view of (33). By (32), $\chi_{11}(t)$ and $\chi_{22}(t)$ are time invariant; thus, $\chi_{11}(t)=\left|d_{1}\right|^{2}=$ const and $\chi_{22}(t)=\left|d_{2}\right|^{2}=$ const for all $t$. For the off-diagonal elements we have $\chi_{12}(t)=\chi_{21}^{*}(t)$; by use of (32) with (21) we obtain

$$
\begin{aligned}
\chi_{12}(t) & =d_{1} d_{2}^{*}+\int_{-\infty}^{t} \mathrm{~d} t^{\prime} \dot{\chi}_{12}\left(t^{\prime}\right) \\
& =d_{1} d_{2}^{*}-2 \mathrm{i} g_{1} \int_{-\infty}^{t} \mathrm{~d} t^{\prime} \Phi_{1}\left(0, t^{\prime}\right) \Phi_{2}^{*}\left(0, t^{\prime}\right)=d_{1} d_{2}^{*}[1-\mathrm{i} \gamma(t)],
\end{aligned}
$$

where

$$
\gamma(t):=\frac{g_{1}}{\pi} \int_{-\infty}^{t} \mathrm{~d} t^{\prime}\left(\int_{0}^{\infty} \mathrm{d} \omega \mathrm{e}^{-\mathrm{i} \omega t^{\prime}} f(\omega)\left[1+S_{1}(\omega)\right]\right)\left(\int_{0}^{\infty} \mathrm{d} \omega^{\prime} \mathrm{e}^{\mathrm{i} \omega^{\prime} t^{\prime}} f^{*}\left(\omega^{\prime}\right)\left[1+S_{2}\left(\omega^{\prime}\right)\right]\right) .
$$

As $t \rightarrow \infty$ the integration over $t^{\prime}$ is carried out explicitly and yields

$$
\begin{aligned}
\lim _{t \rightarrow \infty} \gamma(t) & =2 g_{1} \int_{0}^{\infty} \mathrm{d} \omega|f(\omega)|^{2}\left[1+S_{1}(\omega)\right]\left[1+S_{2}^{*}(\omega)\right] \\
& =8 g_{1} \int_{0}^{\infty} \mathrm{d} \omega|f(\omega)|^{2}\left(1+\frac{\mathrm{i} g_{1}}{2 \sqrt{\omega}}\right)^{-2}=: \gamma_{\infty} .
\end{aligned}
$$

Equations (34) and (36) combined determine $\chi_{12}(t)$ in the limit $t \rightarrow \infty$. 
Next, we derive a formula for $\operatorname{Imp}(M)$ by (30), noting that

$$
\operatorname{det}[\chi(t)]=\chi_{11}(t) \chi_{22}(t)-\chi_{12}(t) \chi_{21}(t)=\left|d_{1} d_{2}\right|^{2}-\left|\chi_{12}(t)\right|^{2} .
$$

The use of (34) entails

$$
\begin{aligned}
\operatorname{Imp}(M) & =2^{1 / 2}\left|d_{1} d_{2}\right|\left[1-\left|1-\mathrm{i} \gamma_{\infty}\right|^{2}\right]^{1 / 2} \\
& =2^{-1 / 2}\left|c_{1}^{2}-c_{2}^{2}\right|\left[1-\left|1-\mathrm{i} \gamma_{\infty}\right|^{2}\right]^{1 / 2},
\end{aligned}
$$

where $\gamma_{\infty}$ is given by (36). Equation (38) is the desired formula for the memory impurity.

To evaluate the impurity for examples to be presented in section 3 , it is convenient to evade the subsidiary condition (28); for this purpose, notice that any nonzero, square integrable function $f$ can be converted into a function that satisfies (28) by dividing $|f|^{2}$ by $4 \int_{0}^{\infty} \mathrm{d} \omega \sqrt{\omega}|f(\omega)|^{2}$. Hence, we replace $\gamma_{\infty}$ of (36) by

$$
\bar{\gamma}_{\infty}:=\frac{2 g_{1} \int_{0}^{\infty} \mathrm{d} \omega|f(\omega)|^{2}\left(1+\frac{\mathrm{i} g_{1}}{2 \sqrt{\omega}}\right)^{-2}}{\int_{0}^{\infty} \mathrm{d} \omega \sqrt{\omega}|f(\omega)|^{2}} .
$$

With this definition, $\bar{\gamma}_{\infty}$ takes the same values as $\gamma_{\infty}$ but represents a different functional of $f$ : the domain of $\bar{\gamma}_{\infty}$ is enlarged to include unnormalized $f$. Thus, we define impurity regardless of whether $f$ is normalized by

$$
\overline{\operatorname{Imp}}(M):=2^{-1 / 2}\left|c_{1}^{2}-c_{2}^{2}\right|\left[1-\left|1-\mathrm{i} \bar{\gamma}_{\infty}\right|^{2}\right]^{1 / 2} .
$$

\subsection{Antisymmetric case}

Next, we apply the steps of section 2.1 to an antisymmetric wavefunction, $\Psi \equiv \Psi_{\text {a }}$; i.e., we solve (15) with (17), and derive a formula for the impurity $\operatorname{Imp}(M)$ in terms of the Fourier transform of $\psi^{\text {in }}(0, t)$.

First, we find $\Psi_{\mathrm{a}}(x, t)$. The Fourier transform in time of (15) yields

$$
\begin{aligned}
& \Psi_{\mathrm{a} j}(x, t)=c_{j} \int_{0}^{\infty} \frac{\mathrm{d} \omega}{\sqrt{2 \pi}} \mathrm{e}^{-\mathrm{i} \omega t} f(\omega)\left[\mathrm{e}^{-\mathrm{i} \sqrt{\omega}|x|}+S_{j}(\omega) \mathrm{e}^{\mathrm{i} \sqrt{\omega}|x|}\right] \operatorname{sg} x, \\
& S_{j}(\omega):=\frac{g_{3 j} \sqrt{\omega}+2 \mathrm{i}}{g_{3 j} \sqrt{\omega}-2 \mathrm{i}}, \quad j=1,2,
\end{aligned}
$$

where $\operatorname{sg} x$ is the sign function, $\operatorname{sg} x:=1$ for $x>0$ and $\operatorname{sg} x:=-1$ for $x<0$, and $f(\omega)$ is arbitrary except for (28); see the appendix for a derivation of (41) and (42). In the limit $t \rightarrow-\infty$, (41) leads to (17) with

$$
\psi^{\text {in }}(x, t) \equiv \int_{0}^{\infty} \frac{\mathrm{d} \omega}{\sqrt{2 \pi}} \mathrm{e}^{-\mathrm{i} \omega t} f(\omega) \mathrm{e}^{-\mathrm{i} \sqrt{\omega}|x|} \operatorname{sg} x .
$$

Condition (28) for $f(\omega)$ then follows by the procedure of section 2.1, which we omit here.

We now express the impurity, $\operatorname{Imp}(M)$, in terms of $f(\omega)$ still using (30) with

$$
\chi(t):=\int_{-\infty}^{\infty} \mathrm{d} x \Psi_{\mathrm{a}}(x, t) \Psi_{\mathrm{a}}^{\dagger}(x, t) .
$$

By analogy with section 2.1 the task is to calculate $\operatorname{det}[\chi(t)]$ as $t \rightarrow \infty$. Differentiation of both sides of (44) in view of (10) furnishes

$$
\dot{\chi}_{j k}(t)=\mathrm{i}\left(g_{3 k}-g_{3 j}\right) \Psi_{\mathrm{a} j}^{\prime}(0, t) \Psi_{\mathrm{a} k}^{\prime *}(0, t), \quad j, k=1,2,
$$


where $g_{3 j}$ are defined by (16); compare with (32). By (17) and (24), $\operatorname{det}[\chi(t)]$ vanishes as $t \rightarrow-\infty$; in particular,

$$
\lim _{t \rightarrow-\infty} \chi(t)=\left[\begin{array}{l}
c_{1} \\
c_{2}
\end{array}\right]\left[c_{1}^{*}, c_{2}^{*}\right]
$$

In view of (45) and (46), $\chi_{11}(t)=\left|c_{1}\right|^{2}$ and $\chi_{22}(t)=\left|c_{2}\right|^{2}$ for all $t$. In addition, we have

$$
\begin{gathered}
\chi_{12}(t)=c_{1} c_{2}^{*}-2 \mathrm{i} g_{3} \int_{-\infty}^{t} \mathrm{~d} t^{\prime} \Psi_{\mathrm{a} 1}^{\prime}\left(0, t^{\prime}\right) \Psi_{\mathrm{a} 2}^{\prime *}\left(0, t^{\prime}\right)=c_{1} c_{2}^{*}[1-\mathrm{i} \gamma(t)], \\
\gamma(t):=\frac{g_{3}}{\pi} \int_{-\infty}^{t} \mathrm{~d} t^{\prime}\left(\int_{0}^{\infty} \mathrm{d} \omega \mathrm{e}^{-\mathrm{i} \omega t^{\prime}} f(\omega) \sqrt{\omega}\left[1+S_{1}(\omega)\right]\right) \\
\times\left(\int_{0}^{\infty} \mathrm{d} \omega^{\prime} \mathrm{e}^{\mathrm{i} \omega^{\prime} t^{\prime}} f^{*}\left(\omega^{\prime}\right) \sqrt{\omega^{\prime}}\left[1+S_{2}^{*}\left(\omega^{\prime}\right)\right]\right) .
\end{gathered}
$$

In particular,

$$
\begin{aligned}
\lim _{t \rightarrow \infty} \gamma(t) & =2 g_{3} \int_{0}^{\infty} \mathrm{d} \omega|f(\omega)|^{2} \omega\left[1+S_{1}(\omega)\right]\left[1+S_{2}^{*}(\omega)\right] \\
& =8 g_{3} \int_{0}^{\infty} \mathrm{d} \omega|f(\omega)|^{2} \omega\left(1+\frac{\mathrm{i} g_{3} \sqrt{\omega}}{2}\right)^{-2}=: \gamma_{\infty} .
\end{aligned}
$$

Equations (47) and (49) determine $\chi_{12}(t)$ in the limit $t \rightarrow \infty$. By (30) we obtain

$$
\operatorname{Imp}(M)=2^{1 / 2}\left|c_{1} c_{2}\right|\left[1-\left|1-\mathrm{i} \gamma_{\infty}\right|^{2}\right]^{1 / 2}
$$

where $\gamma_{\infty}$ is given by (49). By comparison to (38) for symmetric wavefunctions, we infer that the antisymmetric case is not essentially different.

By analogy with section 2.1, for convenience we may define

$$
\bar{\gamma}_{\infty}:=\frac{2 g_{3} \int_{0}^{\infty} \mathrm{d} \omega|f(\omega)|^{2} \omega\left(1+\frac{\mathrm{i} g_{3} \sqrt{\omega}}{2}\right)^{-2}}{\int_{0}^{\infty} \mathrm{d} \omega \sqrt{\omega}|f(\omega)|^{2}} .
$$

The corresponding, renormalized impurity is

$$
\overline{\operatorname{Imp}}(M):=2^{1 / 2}\left|c_{1} c_{2}\right|\left[1-\left|1-\mathrm{i} \bar{\gamma}_{\infty}\right|^{2}\right]^{1 / 2} .
$$

\section{Examples of narrow-band wavefunctions}

In this section we apply the formulation of section 2 to incoming pulse wavefunctions $\psi^{\text {in }}(0, t)$ that are modulated by a carrier frequency $\omega_{0}$ and have a sufficiently narrow frequency spectrum. We discuss the symmetric and antisymmetric cases; and we study three examples of symmetric input pulses by comparing the associated impurities.

We assume that $|f(\omega)|^{2}$ is (i) sharply peaked at $\omega=\omega_{0}>0$, approaching zero fast as $\left|\omega-\omega_{0}\right| / \omega_{0}$ increases; (ii) symmetric about $\omega=\omega_{0}$; and (iii) exponentially negligible on the half line $\omega<0$. Assumptions (i) and (iii) are interrelated and consistent with our consideration of only positive frequencies in the previous section. By (28), (36) and (49), the requisite integrals have the form

$$
\hat{F}[h]:=\int_{0}^{\infty} \mathrm{d} \omega|f(\omega)|^{2} h(\omega),
$$

where $\hat{F}[h]$ denotes a functional of $h$ (given $f$ ); for our purposes, $h(\omega)=\sqrt{\omega},[1+$ $\left.\mathrm{i} g_{1} /(2 \sqrt{\omega})\right]^{-2}$, or $\omega\left(1+\mathrm{i} g_{3} \sqrt{\omega} / 2\right)^{-2}$. Under assumptions (i) and (ii) we expand $\hat{F}[h]$ by

$$
\hat{F}[h] \sim h\left(\omega_{0}\right) \hat{F}[1]+\left.\frac{1}{2} \frac{\mathrm{d}^{2} h}{\mathrm{~d} \omega^{2}}\right|_{\omega=\omega_{0}} \hat{F}\left[\left(\omega-\omega_{0}\right)^{2}\right],
$$

treating $h(\omega)$ and $|f(\omega)|^{2}$ as slowly and rapidly varying, respectively; $\omega_{0}$ is sufficiently large. 


\subsection{Symmetric case}

Following section 2.1 for a symmetric $\psi^{\text {in }}(x, t)$ and using (54) we apply the expansions

$$
\begin{aligned}
& \hat{F}[\sqrt{\omega}] \sim \sqrt{\omega_{0}} \hat{F}[1]-\left(2 \sqrt{\omega_{0}}\right)^{-3} \hat{F}\left[\left(\omega-\omega_{0}\right)^{2}\right], \\
& \hat{F}\left[\left(1+\frac{\mathrm{i} g_{1}}{2 \sqrt{\omega}}\right)^{-2}\right] \sim(1+\mathrm{i} \eta)^{-2} \hat{F}[1]-\frac{3 i \eta}{4 \omega_{0}^{2}}(1+\mathrm{i} \eta)^{-4} \hat{F}\left[\left(\omega-\omega_{0}\right)^{2}\right],
\end{aligned}
$$

where

$$
\eta:=\frac{g_{1}}{2 \sqrt{\omega_{0}}} .
$$

The substitution of (55) and (56) into (39) gives

$$
\bar{\gamma}_{\infty}=\frac{4 \eta}{(1+i \eta)^{2}}\left\{1+\frac{\hat{F}\left[\left(\omega-\omega_{0}\right)^{2}\right]}{8 \omega_{0}^{2} \hat{F}[1]}\left[1-\frac{6 i \eta}{(1+i \eta)^{2}}\right]\right\}+O\left(\omega_{0}^{-4}\right),
$$

by which (40) becomes

$$
\overline{\operatorname{Imp}}(M) \sim \sqrt{2}\left|c_{1}^{2}-c_{2}^{2}\right| \frac{|\eta|}{1+\eta^{2}} \frac{1}{\omega_{0}}\left(\frac{\hat{F}\left[\left(\omega-\omega_{0}\right)^{2}\right]}{\hat{F}[1]}\right)^{1 / 2} .
$$

In the next paragraph this expression is evaluated for three cases of input pulses.

Example 1 (Single-frequency incoming pulse). For the limit of a single-frequency incoming wavefunction we take

$$
|f(\omega)|^{2} \longrightarrow \frac{1}{4 \sqrt{\omega_{0}}} \delta\left(\omega-\omega_{0}\right),
$$

where the factor in the right-hand side of this equation is chosen to conform to (28). Thus, $\hat{F}\left[\left(\omega-\omega_{0}\right)^{2}\right] \longrightarrow 0$; by $(59)$,

$$
\overline{\operatorname{Imp}}(M) \longrightarrow 0 .
$$

This limit is expected; it corresponds to Wu's [9] admissible incoming wavefunction.

The reason for the square root in the definition (3) for impurity can now be explained briefly, by examining for this case with zero impurity the change in the memory state brought about by the particle. As a measure of this change we take the angle $\theta:=$ $\cos ^{-1}\left|\left\langle c^{\text {in }} \mid c^{\text {out }}\right\rangle\right|$ between the memory 'in-state' $\left|c^{\text {in }}\right\rangle=\left[c_{1}, c_{2}\right]^{\mathrm{T}}$ and 'out-state' $\left|c^{\text {out }}\right\rangle$, which are vectors representing the state of the memory initially $(t \rightarrow-\infty)$ and finally $(t \rightarrow \infty)$. Equations (20)-(22) and (60) assert that $\left|c^{\text {in }}\right\rangle$ is related to $\left|c^{\text {out }}\right\rangle$ by a unitary transformation. Accordingly,

$$
\begin{aligned}
\left\langle c^{\text {in }} \mid c^{\text {out }}\right\rangle= & \left\langle d^{\text {in }} \mid d^{\text {out }}\right\rangle=\left[d_{1}^{*}, d_{2}^{*}\right]\left[\begin{array}{cc}
S_{1}\left(\omega_{0}\right) & 0 \\
0 & S_{2}\left(\omega_{0}\right)
\end{array}\right]\left[\begin{array}{l}
d_{1} \\
d_{2}
\end{array}\right] \\
= & \left(\frac{1-\mathrm{i} \eta}{1+\mathrm{i} \eta}\right)\left|d_{1}\right|^{2}+\left(\frac{1+\mathrm{i} \eta}{1-\mathrm{i} \eta}\right)\left|d_{2}\right|^{2}, \\
\left|\left\langle c^{\text {in }} \mid c^{\text {out }}\right\rangle\right|^{2} & =\left[\left(1-\eta^{2}\right)^{2}+4 \eta^{2}\left(\left|d_{2}\right|^{2}-\left|d_{1}\right|^{2}\right)^{2}\right] /\left(1+\eta^{2}\right)^{2} \\
& =1-\frac{16 \eta^{2}\left|d_{1} d_{2}\right|^{2}}{\left(1+\eta^{2}\right)^{2}}=1-\frac{4 \eta^{2}\left|c_{1}^{2}-c_{2}^{2}\right|^{2}}{\left(1+\eta^{2}\right)^{2}} .
\end{aligned}
$$


Thus, we find that

$$
|\sin \theta|=\frac{2|\eta|\left|c_{1}^{2}-c_{2}^{2}\right|}{1+\eta^{2}} .
$$

A comparison of this formula to (59) shows the desired scaling property mentioned in the Introduction: with our definition (3) for $\operatorname{Imp}(M)$, the ratio of $\operatorname{Imp}(M)$ to $|\sin \theta|$ is independent of both the starting state, $\left[c_{1}, c_{2}\right]^{\mathrm{T}}$, and the coupling constant, $g_{1}$.

Example 2 (Gaussian envelope). Next, we consider an unnormalized $\psi^{\text {in }}(0, t)$ with a Gaussian envelope of width $T$, i.e.,

$$
\psi^{\text {in }}(0, t) \sim \mathrm{e}^{-t^{2} /\left(2 T^{2}\right)} \mathrm{e}^{-\mathrm{i} \omega_{0} t}
$$

where corrections to $\psi^{\text {in }}(0, t)$ due to the omission of negative frequencies are left out. The Fourier transform in $t$ of this $\psi^{\text {in }}(0, t)$ is

$$
f(\omega) \sim \exp \left[-\left(\omega-\omega_{0}\right)^{2} T^{2} / 2\right],
$$

where $\omega_{0} T \gg 1$ so that the negative-frequency part is negligible. It follows by (53) that $\hat{F}[1]=\sqrt{\pi} / T$, and $\hat{F}\left[\left(\omega-\omega_{0}\right)^{2}\right]=\sqrt{\pi} /\left(4 T^{3}\right)$; thus, by (59) we find

$$
\overline{\operatorname{Imp}}(M)=\frac{|\sin \theta|}{2 \omega_{0} T},
$$

where $|\sin \theta|$ is defined by (64). Equation (67) asserts that reducing the impurity, while maintaining the angle, $\theta$, between in and out memory states, can be achieved only by increasing $\omega_{0} T$; more precisely, the product of $\operatorname{Imp}(M)$ and $\omega_{0} T$ is fixed.

Example 3 (Convolution of rectangular and Gaussian envelopes). Here we analyse an incoming wavefunction $\psi^{\text {in }}(0, t)$ with an envelope that results by convoluting a rectangular pulse of length $2 T$ with the Gaussian shape $\exp \left[-t^{2} /\left(2 \tau^{2}\right)\right]$; the convolution is introduced to cause smoothing. This case corresponds closely to a $\psi^{\mathrm{in}}(0, t)$ that is switched on at $t=-T$ and switched off at $t=T$. We find that $\psi^{\text {in }}(0, t)$ is proportional to $\mathrm{e}^{-\mathrm{i} \omega_{0} t}\{\operatorname{erf}[(t+T) /(\sqrt{2} \tau)]-\operatorname{erf}[(t-T) /(\sqrt{2} \tau)]\}$ (apart from a small correction due to the exclusion of negative frequencies); $\operatorname{erf}(z):=2 \pi^{-1 / 2} \int_{0}^{z} \mathrm{~d} s \mathrm{e}^{-s^{2}}$. The Fourier transform of this $\psi^{\text {in }}(0, t)$ is

$$
\begin{aligned}
f(\omega) & \sim \int_{-\infty}^{\infty} \frac{\mathrm{d} t}{\sqrt{2 \pi}} \mathrm{e}^{\mathrm{i}\left(\omega-\omega_{0}\right) t}\left[\operatorname{erf}\left(\frac{t+T}{\sqrt{2} \tau}\right)-\operatorname{erf}\left(\frac{t-T}{\sqrt{2} \tau}\right)\right] \\
& =\exp \left[-\tau^{2}\left(\omega-\omega_{0}\right)^{2} / 2\right] \frac{\sin \left[\left(\omega-\omega_{0}\right) T\right]}{\omega-\omega_{0}} \quad \omega_{0} T \gg 1 .
\end{aligned}
$$

By using integration by parts and integral tables [15], we find

$$
\begin{aligned}
& \hat{F}[1]=\pi T \operatorname{erf}(T / \tau)-\sqrt{\pi} \tau\left[1-\mathrm{e}^{-(T / \tau)^{2}}\right], \\
& \hat{F}\left[\left(\omega-\omega_{0}\right)^{2}\right]=\frac{\sqrt{\pi}}{2 \tau}\left[1-\mathrm{e}^{-(T / \tau)^{2}}\right] .
\end{aligned}
$$

These formulae are simplified for short smoothing time, $\tau \ll T$ so that $\mathrm{e}^{-(T / \tau)^{2}} \approx 0$; accordingly, by (59) the impurity is

$$
\overline{\operatorname{Imp}}(M) \sim\left|c_{1}^{2}-c_{2}^{2}\right| \frac{|\eta|}{1+\eta^{2}} \frac{1}{\omega_{0} \tau^{1 / 2}(\sqrt{\pi} T-\tau)^{1 / 2}},
$$

which is inverse proportional to the square root of the pulse length, $2 T$, when $T \gg \tau$. This behaviour is in marked contrast to the case with a Gaussian envelope in which the impurity decreases inverse linearly to the pulse length; see (67). 


\subsection{Antisymmetric case}

By section 2.2 for an antisymmetric $\psi^{\text {in }}(x, t)$ and (54) we need the additional expansion

$$
\hat{F}\left[\omega\left(1+\frac{\mathrm{i} g_{3} \sqrt{\omega}}{2}\right)^{-2}\right] \sim \frac{\omega_{0}}{(1+\mathrm{i} \eta)^{2}} \hat{F}[1]-\frac{3 \mathrm{i}}{4 \omega_{0}} \frac{\eta}{(1+\mathrm{i} \eta)^{4}} \hat{F}\left[\left(\omega-\omega_{0}\right)^{2}\right]
$$

where

$$
\eta:=\frac{g_{3} \sqrt{\omega_{0}}}{2}
$$

In order to obtain $\bar{\gamma}_{\infty}$ we substitute (55) and (72) into (51). After some algebra we derive (58), i.e., we obtain a formula for $\bar{\gamma}_{\infty}$ that is identical to that of the symmetric case but with definition (73). Consequently, by inspection of (52) and use of (59), we infer that

$$
\overline{\operatorname{Imp}}(M) \sim 2 \sqrt{2}\left|c_{1} c_{2}\right| \frac{|\eta|}{1+\eta^{2}} \frac{1}{\omega_{0}}\left(\frac{\hat{F}\left[\left(\omega-\omega_{0}\right)^{2}\right]}{\hat{F}[1]}\right)^{1 / 2}
$$

Note that for the antisymmetric case $|\sin \theta|=4|\eta|\left|c_{1} c_{2}\right| /\left(1+\eta^{2}\right)$.

\section{Minimization of impurity for given pulse duration}

In this section we formulate the problem of determining the pulse shape that minimizes impurity given a duration of the incident pulse interaction with the memory at $x=0$. For narrow-band pulses we solve this problem to show that the optimal incoming wavefunction has Gaussian envelope.

First, we define the pulse duration, $T$, at $x=0$ via the ratio

$$
T^{2}:=2 \frac{\int_{-\infty}^{\infty} \mathrm{d} t t^{2}\left|\psi^{\mathrm{in}}(0, t)\right|^{2}}{\int_{-\infty}^{\infty} \mathrm{d} t\left|\psi^{\mathrm{in}}(0, t)\right|^{2}}=2 \frac{\int_{0}^{\infty} \mathrm{d} \omega\left|\frac{\mathrm{d} f}{\mathrm{~d} \omega}\right|^{2}}{\int_{0}^{\infty} \mathrm{d} \omega|f(\omega)|^{2}},
$$

where the factor 2 is included for consistency with the Gaussian envelope used in section 3.1. In deriving the second equality in (75) we assume routinely that the integrals converge and apply Parseval's formula [16].

\subsection{Problem statement}

Next, we pose a constrained minimization problem for the memory impurity, $\operatorname{Imp}(M)$, on the basis of (40) for the symmetric case and (52) for the antisymmetric case, by (i) considering pulses with fixed duration, $T$, which is defined by (75); and (ii) properly scaling out the effect of the initial memory state, $\left[c_{1}, c_{2}\right]^{\mathrm{T}}$.

Problem I. Determine the $f(\omega)$ that minimizes $2^{1 / 2} \overline{\operatorname{Imp}}(M) /\left|c_{1}^{2}-c_{2}^{2}\right|$ for the symmetric case, equation (40), or $2^{-1 / 2} \overline{\operatorname{Imp}}(M) /\left|c_{1} c_{2}\right|$ for the antisymmetric case, equation (52), under the constraint of a fixed $T^{2}$ given by (75).

Notably, if $f=\breve{f}(\omega)$ is a solution to problem I, then $K_{f} \cdot \breve{f}$ is also a solution for any nonzero constant $K_{f}$. Bearing this property in mind along with the definition of $\bar{\gamma}_{\infty}$ from (39) or (51), we state an equivalent problem. 
Problem II. Determine the $f(\omega)$ that minimizes the functional $\hat{I}[f]:=2^{1 / 2} \operatorname{Imp}(M) /\left|c_{1}^{2}-c_{2}^{2}\right|$ for the symmetric case, (38), or $\hat{I}[f]=2^{-1 / 2} \operatorname{Imp}(M) /\left|c_{1} c_{2}\right|$ for the antisymmetric case, (50), under the constraint of fixed $\hat{N}_{x}[f]:=\hat{F}[\sqrt{\omega}], \hat{N}_{t}[f]:=\hat{F}[1]$, and

$$
\hat{C}[f]:=\int_{0}^{\infty} \mathrm{d} \omega\left|\frac{\mathrm{d} f}{\mathrm{~d} \omega}\right|^{2} .
$$

In problem II $\hat{F}[h]$ is defined by (53), and the subscripts $x$ and $t$ in $\hat{N}$ denote the origin of the corresponding normalization condition (i.e., integration in space or time); cf (24)-(28) and (75) via Parseval's formula. Because the involved functionals are manifestly convex, problem II admits a meaningful solution, which can be obtained by the method of Lagrange multipliers. Thus, we can consider the unconstrained extremization of $\hat{I}+\mu_{x} \hat{N}_{x}-\mu_{t} \hat{N}_{t}+\mu_{C} \hat{C}$ where $\mu_{l}(l=x, t, C)$ are suitable constants; note in passing that the constraints here are not independent. This procedure leads to a second-order differential equation for $f(\omega)$. Although the derivation and solution of this equation in generality lie beyond the scope of this paper, the minimization problem is simplified considerably and solved for narrow-band wavefunctions, as discussed below.

\subsection{Narrow-band incoming pulse}

We now resort to the formulation of section 3 in order to derive an explicit formula for the optimal $f(\omega)$. By inspection of (59) (symmetric case) or (74) (antisymmetric case) along with definition (75), we state the following simplified problem.

Problem III (for narrow-band pulses). Determine the $f(\omega)$ that minimizes $\hat{I}[f]:=$ $\hat{F}\left[\left(\omega-\omega_{0}\right)^{2}\right]$ with fixed $\hat{N}_{t}[f], \hat{C}[f]$, and given carrier frequency $\omega_{0}$.

This problem is equivalent to describing the ground state of a quantum harmonic oscillator in the $\omega$-space: $\hat{I}$ represents the 'potential energy', $\hat{C}$ is the 'kinetic energy', and $\hat{N}_{t}$ accounts for the wavefunction normalization. Hence, it can be expected that the solution to problem III is a Gaussian.

We now sketch the solution of problem III via Lagrange multipliers, extremizing $\hat{I}_{\text {tot }}[f]=\hat{I}[f]-\mu_{t} \hat{N}_{t}[f]+\mu_{C} \hat{C}[f]$ without constraint. Because the functionals $\hat{I}, \hat{C}$ and $\hat{N}_{t}$ are quadratic and positive definite, the extremization yields a minimum. By setting equal to zero the first variation of $\hat{I}_{\text {tot }}[f]$ with respect to $f^{*}(\omega)$ we obtain

$$
\int_{0}^{\infty} \mathrm{d} \omega\left\{\left[\left(\omega-\omega_{0}\right)^{2}-\mu_{t}\right] f(\omega)-\mu_{C} \frac{\mathrm{d}^{2} f}{\mathrm{~d} \omega^{2}}\right\} \delta f^{*}(\omega)=0,
$$

where $\delta f^{*}$ denotes the variation of $f^{*}$, and we applied integration by parts taking $f(0) \approx 0$ and $f(\omega \rightarrow \infty)=0$. Thus, $f(\omega)$ solves

$$
-\frac{\mathrm{d}^{2} f}{\mathrm{~d} \Omega^{2}}+\Omega^{2} f=\mu f, \quad \Omega:=\mu_{C}^{-1 / 4}\left(\omega-\omega_{0}\right), \quad \mu:=\frac{\mu_{t}}{\sqrt{\mu_{C}}} .
$$

Admissible solutions to this differential equation are [17] $f_{n}(\omega):=H_{n}(\Omega) \mathrm{e}^{-\Omega^{2} / 2}$ with $\mu=$ $2 n+1$, where $H_{n}$ are Hermite polynomials of degree $n=0,1, \ldots$ Thus, $f \equiv f_{0}=\mathrm{e}^{-\Omega^{2} / 2}$ yields an absolute minimum for $\hat{I}$ and is the desired solution. It follows by (75) that $\mu_{C}=T^{-4}$ so that $f(\omega)=\mathrm{e}^{-\left(\omega-\omega_{0}\right)^{2} T^{2} / 2}$. By (67) the impurity for this pulse envelope behaves as $\overline{\operatorname{Imp}}(M)=O\left[\left(\omega_{0} T\right)^{-1}\right]$. 


\section{Conclusion}

We studied the decoherence induced in a quantum memory when its state is transformed by a pulsed beam of nonrelativistic, massive particles incident on the memory in one space dimension. When probed by the pulsed beam, the initially pure memory state becomes entangled with the particle wavefunction. Our main results are: (1) speeding computations by using shorter pulses increases this operation-induced decoherence; and (2) minimal decoherence for given pulse duration is achieved with Gaussian pulse envelopes. By contrast, the decoherence due to extraneous influences [6,7], unconnected to scattering and memory operations, decreases with shorter pulses. Our analysis corroborates the main, general conclusion in [9], which was based on scattering of light pulses by the memory. However, the optimal light pulse in [9] has a half-cycle cosine envelope, which preserves its shape because of the assumed non-dispersive beam propagation in this case.

The impurity by which we express decoherence is also a measure of the entanglement of the outgoing field $\Psi^{\text {out }}$ for the combined particle-memory system. The usual entanglement of formation for this field is just the von Neumann entropy of the reduced density matrix $M$ of the memory [18]. The von-Neumann entropy of $M$ varies monotonically with $\operatorname{Imp}(M)$, so that $\operatorname{Imp}(M)$ serves as an alternative measure of the entanglement of the out field $\Psi^{\text {out }}$. The complementarity of the entanglement of the out field and the possibility of interference effects critical to quantum computing are discussed in [19].

Our assumption of a point interaction enabled the explicit calculation of $\operatorname{Imp}(M)$. However, we expect our result for the incoming pulse envelope that minimizes decoherence to hold for a wide class of localized interaction potentials provided that the pulse duration is sufficiently large. Such a generalization has been achieved [9] in the case of photon scattering from the quantum memory.

Our restriction to one space dimension has application to memories connected to particle sources by narrow wires. However, there remain open problems of studying scattering from the memory in two and three space dimensions.

\section{Acknowledgments}

We thank Tai T Wu and Howard E Brandt for useful discussions.

\section{Appendix. Solutions of Schrödinger's equation with point interaction}

In this appendix we derive formulae (21) and (22) for symmetric wavefunctions, along with (41) and (42) for antisymmetric ones. The Fourier transform of a function $\phi(t)$ is defined by

$$
\tilde{\phi}(\omega):=\int_{-\infty}^{+\infty} \frac{\mathrm{d} t}{\sqrt{2 \pi}} \phi(t) \mathrm{e}^{\mathrm{i} \omega t}
$$

First, we address the symmetric case. By (13), the Fourier transform $\tilde{\Phi}_{j}(x, \omega)$ of each scalar component $\Phi_{j}(x, t)$ satisfies

$$
-\partial_{x x} \tilde{\Phi}_{j}(x, \omega)+g_{1 j} \delta(x) \tilde{\Phi}_{j}(0, \omega)=\omega \tilde{\Phi}_{j}(x, \omega),
$$

where $\tilde{\Phi}_{j}(x, \omega)=\tilde{\Phi}_{j}(-x, \omega)$ from (11) with $\Psi=\Psi_{\mathrm{s}}$. Thus, the solution of (A.2) is

$$
\tilde{\Phi}_{j}(x, \omega)=C(\omega) \mathrm{e}^{-\mathrm{i} \sqrt{\omega}|x|}+K(\omega) \mathrm{e}^{\mathrm{i} \sqrt{\omega}|x|},
$$


where $C$ and $K$ are to be found. By direct differentiation of (A.3) we obtain

$$
\begin{aligned}
& \partial_{x} \tilde{\Phi}_{j}=\mathrm{i} \sqrt{\omega}\left(-C \mathrm{e}^{-\mathrm{i} \sqrt{\omega}|x|}+K \mathrm{e}^{\mathrm{i} \sqrt{\omega}|x|}\right) \operatorname{sg} x, \\
& \partial_{x x} \tilde{\Phi}_{j}=2 \mathrm{i} \sqrt{\omega}(-C+K)-\omega\left(C \mathrm{e}^{-\mathrm{i} \sqrt{\omega}|x|}+K \mathrm{e}^{\mathrm{i} \sqrt{\omega}|x|}\right) \delta(x),
\end{aligned}
$$

where $\operatorname{sg} x$ is the sign function ( $\operatorname{sg} x:=1$ if $x>0$ and $\operatorname{sg} x:=-1$ if $x<0), \partial_{x}|x|=\operatorname{sg} x$ and $\partial_{x} \operatorname{sg} x=2 \delta(x)$. The substitution of (A.3) and (A.5) into (A.2) yields

$$
K=\frac{2 \mathrm{i} \sqrt{\omega}+g_{1 j}}{2 \mathrm{i} \sqrt{\omega}-g_{1 j}} C .
$$

Equations (21) and (22) follow by setting $C(\omega) \equiv d_{j} f(\omega)$.

Next, we address the antisymmetric case starting with $(15) ; \delta_{p}^{\prime}(x)=\delta^{\prime}(x)$ and $\Psi_{\mathrm{a} j}^{\prime}(0, t)=\lim _{x \rightarrow 0^{ \pm}} \partial_{x} \Psi_{\mathrm{a} j}(x, t)$. The Fourier transform $\widetilde{\Psi}_{\mathrm{a} j}(x, \omega)$ of $\Psi_{\mathrm{a} j}(x, t)$ satisfies

$$
\partial_{x x} \tilde{\Psi}_{\mathrm{a} j}(x, \omega)-g_{3 j} \delta^{\prime}(x) \tilde{\Psi}_{\mathrm{a} j}^{\prime}(0, \omega)=\omega \tilde{\Psi}_{\mathrm{a} j}(x, \omega) .
$$

Because $\tilde{\Psi}_{\mathrm{a} j}(x, \omega)=-\tilde{\Psi}_{\mathrm{a} j}(-x, \omega)$, the solution to (A.7) is

$$
\tilde{\Psi}_{\mathrm{a} j}(x, \omega)=\left[C(\omega) \mathrm{e}^{-\mathrm{i} \sqrt{\omega}|x|}+K(\omega) \mathrm{e}^{\mathrm{i} \sqrt{\omega}|x|}\right] \operatorname{sg} x .
$$

By successive differentiations of (A.8) we obtain

$$
\begin{gathered}
\partial_{x} \tilde{\Psi}_{\mathrm{a} j}=2 \delta(x)\left(C \mathrm{e}^{-\mathrm{i} \sqrt{\omega}|x|}+K \mathrm{e}^{\mathrm{i} \sqrt{\omega}|x|}\right)+\mathrm{i} \sqrt{\omega}\left(-C \mathrm{e}^{-\mathrm{i} \sqrt{\omega}|x|}+K \mathrm{e}^{\mathrm{i} \sqrt{\omega}|x|}\right) \\
\quad=2(C+K) \delta(x)+\mathrm{i} \sqrt{\omega}\left(-C \mathrm{e}^{-\mathrm{i} \sqrt{\omega}|x|}+K \mathrm{e}^{\mathrm{i} \sqrt{\omega}|x|}\right) \\
\partial_{x x} \tilde{\Psi}_{\mathrm{a} j}(x, \omega)=2(C+K) \delta^{\prime}(x)-\omega\left(C \mathrm{e}^{-\mathrm{i} \sqrt{\omega}|x|}+K \mathrm{e}^{\mathrm{i} \sqrt{\omega}|x|}\right) \operatorname{sg} x
\end{gathered}
$$

using $(\operatorname{sg} x)^{2}=1$. The substitution of (A.8) and (A.10) into (A.7) yields

$$
K=\frac{g_{3 j} \sqrt{\omega}+2 \mathrm{i}}{g_{3 j} \sqrt{\omega}-2 \mathrm{i}} C .
$$

Equations (41) and (42) are recovered via $C(\omega) \equiv c_{j} f(\omega)$.

\section{References}

[1] Benioff P 1980 J. Stat. Phys. 22563

[2] Albert D Z 1983 Phys. Lett. A 98249

[3] Feynman R P 1985 Opt. News 1111

[4] Deutch D 1985 Proc. R. Soc. A 40097

[5] Shor P W 1995 Phys. Rev. A 52 R2493

[6] Nielsen M A and Chuang I L 2000 Quantum Computation and Quantum Information (Cambridge: Cambridge University Press)

[7] Jaeger G 2006 Quantum Information: An Overview (Berlin: Springer)

[8] Wu T T and Yu M L 2002 J. Math. Phys. 435949

[9] Wu T T 2003 Quantum Information and Computation ed E Donkor, A R Pirich and HE Brandt (Bellingham, WA: SPIE) Proc. SPIE 5105204 (http://spiedl.aip.org/getabs/servlet/GetabsServlet?prog=normal\&id=PSISDG $005105000001000204000001 \&$ idtype $=$ cvips $\&$ gifs $=$ Yes \&bproc $=$ volrange $\&$ scode $=5100 \% 20-\% 205199)$

[10] Wu T T 2004 Quantum Information and Computation II ed E Donkor, A R Pirich and H E Brandt (Bellingham, WA: SPIE) Proc. SPIE 5436 81(http://spiedl.aip.org/getabs/servlet/GetabsServlet?prog=normal\&id=PSISDG $005436000001000081000001 \&$ idtype $=$ cvips $\&$ gifs $=$ Yes $\&$ bproc $=$ volrange $\&$ scode $=5400 \% 20-\% 205499$ )

[11] Wu T T 2005 Quantum Information and Computation III ed E Donkor, A R Pirich and H E Brandt (Bellingham, WA: SPIE) Proc. SPIE 581562 (http://spiedl.aip.org/getabs/servlet/GetabsServlet?prog=normal\&id=PSISDG $005815000001000062000001 \&$ idtype $=$ cvips $\&$ gifs $=$ Yes $\&$ bproc $=$ volrange $\&$ scode $=5800 \% 20-\% 205899$ ) 
[12] Khuri N N and Wu T T 1997 Phys. Rev. D 566779

Khuri N N and Wu T T 1997 Phys. Rev. D 566785

[13] Barnes J P and Warren W S 1999 Phys. Rev. A 604363

[14] Braunstein S L and Loock van P 2005 Rev. Mod. Phys. 77513

[15] Gradshteyn I S and Ryzhik I M 2000 Tables of Integrals, Series, and Products (San Diego, CA: Academic)

[16] Dym H and McKean H P 1972 Fourier Series and Integrals (New York: Academic)

[17] Bateman Manuscript Project 1953 Higher Transcendental Functions ed A Erdélyi (New York: McGraw-Hill) vol II p 117

[18] Wootters W K 1998 Phys. Rev. Lett. 802245

[19] Jaeger G, Horne M A and Shimony A 1993 Phys. Rev. A 481023

Jaeger G, Shimony A and Vaidman L 1995 Phys. Rev. A 5154 